\title{
MJN LEVEL OF ANXIETY, FATIGUE AND DEPRESSION AMONG COLLEGE GRADUATING STUDENTS ENROLLED IN BILIRAN PROVINCE STATE UNIVERSITY
}

\author{
Pearl Irish V. De Paz*, Tracy D. Armstrong, Richelle Ann F. Mullon \\ Biliran State University, School of Nursing and Health Sciences-Main Campus, Naval, Biliran, Philippines \\ *Corresponding Author's Email: pearlirish.depaz@bipsu.edu.ph
}

\begin{abstract}
Background: The need to finish the graduating degree becomes very compelling to the students to meet the expectations of their families, relatives and friends. However, when the student faces obstacles to meet the said expectations, the student may experience emotional problems such as fatigue, anxiety and depression especially among those who are about to finish the degree. Assessment of the student's mental health, specifically the level of fatigue, anxiety and depression is vital to prevent further complications. The purpose of this study is to determine the prevalence of anxiety, fatigue and depression among graduating students enrolled at Biliran Province State University. Methods: The study used a descriptive survey design using standardized tools such as the Beck's Anxiety Inventory (BDI), Brief Fatigue Inventory (BFI) and the Kutcher's Adolescent Depression Scale to determine the prevalence of anxiety, fatigue and depression among the respondents. Results: The results revealed that most of the respondents had mild anxiety, fatigue and depression. However, there were also some students who experienced moderate-severe anxiety, fatigue and depression. Conclusion: The results of the study highlight the need to craft interventions such as health education campaigns or counselling that will help students in the prevention of anxiety, fatigue and depression especially among graduating students.
\end{abstract}

Keywords: Anxiety; Fatigue; Depression; Graduating Students; Mental Health Status

\section{INTRODUCTION}

Anxiety, fatigue and depression are considered to be the common mental health problems faced by students especially before graduation. This may be due to several factors or stressors such as academic pressures, family and financial problems Pedrelli et al., 2015. Moreover, the need to finish the degree becomes compelling because of the expectations of the student's family, relatives and friends. Thi because having a degree is a primary requirement for being employed (Lee et al., 2013). Hence, when the student is not able to meet the said expectations, mental health problems such as anxiety, fatigue that may even lead to depression may arise.

Anxiety is described as the feeling of being tensed or nervous and with the physical changes like elevated blood pressure, distress, uneasiness. On the other hand, fatigue is also defined as likely to be weak, tired, dozy, and the physical and mental state of being extremely worn-out while depression is when a person feels unhappy, down casted and uninterested in performing activities of daily living. Furthermore, literature suggests that anxiety and fatigue are usually experienced by individuals who encountered stressors or difficulties. However, when fatigue and anxiety are prolonged, left untreated or managed, it may lead to depression. (American Psychiatric Association, 2013).

Moreover, studies also show that during young adulthood, most mental health problems occur during young adulthood (Kessler et al., 2007). Approximately $11.9 \%$ of the college students are suffering from an anxiety disorder (Blanco et al., 2008), 55.9\% of students experienced fatigue (Soyeur et al., 2010) while $7-9 \%$ of college students experienced depression most 
mental health problems arise among young adults (Kessler et al., 2007). Furthermore, studies show that $11.9 \%$ of the college students are suffering from an anxiety disorder (Blanco et al., 2008), while $55.9 \%$ of students experienced fatigue (Soyeur et al., 2010) 7-9\% of college students experienced depression (Eisenberg et al., 2013). Several studies have been conducted outside the country on the prevalence of anxiety, fatigue and depression among college students. However, limited studies were conducted in the Philippines, which focused on the assessment of anxiety, fatigue and depression especially among graduating students. Hence, the study was conducted. The results of the study will serve as a guide in crafting interventions to decrease or prevent the prevalence of the said mental health problems.

\section{PURPOSE}

The current study sought to determine levels of anxiety, fatigue and depression among college graduating students enrolled in Biliran Province State University.

\section{METHODOLOGY}

\section{Study Design, Setting and Participants}

The study utilized the descriptive-survey design using three standardized tools such as the 21-item Beck Anxiety Inventory, 9-item Brief Fatigue Inventory and the 11-ITEM Kutcher Adolescent Depression Scale for the data collection. The study aimed to determine the prevalence of anxiety, fatigue and depression among graduating college students in Biliran Province State University.

The respondents of the study were the graduating college students enrolled at Biliran Province State University. A total of 50 respondents coming from the colleges of the Arts and Sciences, Education, Industrial Information and Computer Technology and 30 students in College of Engineering with a total of 230 students were utilized as the respondents using purposive sampling. Moreover the participants using the following criteria: (1) Must be 18 years old and above; (2) He or she must be officially enrolled for the year 2018-2019; (3) Must be a regular student which means he or she must have a full academic load of the year for the level enrolled; (4) Must be a graduating student on March 2019 ; (5) He or she voluntarily decided to participate in the study. The exclusion criteria are those not mentioned in the inclusion criteria and those students who refused to be part of the study were excluded.

\section{Data Collection Procedure}

First, transmittal letters were sent to the concerned offices such as to the Office of the University President, Research Services, deans and chairpersons in order to ask for the permission and approval to conduct the study. Informed consents were secured by asking the respondents to sign the consent form which indicated the respondent's voluntary decision to be part of study. Likewise, the purpose of the study and the questionnaire were comprehensively explained among the respondents. Next, the standardized questionnaires were distributed among the participants and the accomplished questionnaires were then tallied, analyzed, presented in tabular forms and interpreted.

\section{Data Collection Instrument}

The study used three standardized tools for data gathering namely the Beck Anxiety Inventory (Beck et al., 1988), Brief Fatigue Inventory (University of Texas MD Anderson Cancer Center., 1997) and the Kutcher's Adolescent Depression Scale or KADS-11 (LeBlanc et al., 2002). The first part of the questionnaire pertains to 20 statements which indicated the signs and symptoms of anxiety to determine the level of anxiety which is answerable by a Likert's scale ranging from $0-3$ wherein 0 is not at all, 1 is mildly but did not bothered much, 2 is moderately, and 3 is severely and it bothered them much. On the other hand, the second part answered the questions on determining the prevalence of fatigue. The tool pertains to 9 statements which indicated the signs and symptoms of fatigue, which is answered by a score of $0-10$ while the last part of the questionnaire pertains to 11 statements which indicated the signs and symptoms of depression which is answered by a Likert's Scale of 0-3.

\section{Statistical Analysis}

The data gathered were tallied accordingly and was compared with the required data and plotted and graphed thereupon for the analysis of data. The data were described using the frequency and percentage formula. The data on the prevalence of anxiety was generated from the 21-item Beck Anxiety Inventory which pertains to 20 statements that indicated the signs and symptoms of anxiety which is answerable by $0-3$. Meanwhile, the level of fatigue was derived from the 9-item Brief Fatigue Inventory which refers to the 9 statements that indicated the signs and symptoms of fatigue which is answerable by $0-3$ while the severity of depression was derived from the 11-item Kutcher Adolescent Depression Scale which 
pertains to 11 statements that showed the signs and symptoms of depression and is answerable by a $0-10$. The data were analyzed based on the computed scores of the participants. A total score of 0-21 indicates that anxiety is mild, 22-35 means anxiety is moderate, and a score of 36 and more indicates that anxiety is severe. Moreover, the prevalence of fatigue was also determined using the scoring directions of Brief Fatigue Inventory ranging from $0-90$. A total score of $0-30$ is indicative of mild fatigue, 31-60 is moderate fatigue and $61-90$ is considered as severe fatigue. On the other hand, the prevalence of depression was determined using the scoring direction of the Kutcher's Adolescent Depression scale. A total score of 0-7 is indicative that the person has no depression, 8-16 means that depression is mild, 17-23 means that depression is moderate and a score of 24 up indicates that the depression is severe. Lastly, the overall level of anxiety, fatigue and depression were determined using the measure of central tendency known as mean.

\section{Ethical Considerations}

Before the conduct of the study, the proposal was first submitted for the approval of the University Ethics Committee. Furthermore, the respondents of the study were provided with informed consents indicating the purpose of the research, the potential benefits and harm of the research towards them and ensure their complete anonymity throughout the research. The researcher addressed issues such as honesty, objectivity, respect, for intellectual properly, social responsibility, privacy, non-discrimination and others. Furthermore, answers to completed questionnaires were secured and stored in a secured place. After which, all results of the study will be kept for one year and will be burned afterwards. The ethical aspect of research was followed strictly in the contract of this research.

\section{RESULTS}

A total of 230 graduating students from Biliran Province State University agreed to participate in the study and completed the questionnaire. Table 1 shows the anxiety level while Table II shows the quality fatigue level and Table 3 shows the depression level of the respondents.

\section{Level of Anxiety}

As illustrated in Table 1, the majority of the respondents experienced a mild level of anxiety $(59.67 \%)$ followed by moderate anxiety $(24.78 \%)$ and severe anxiety $(15.85 \%)$.
Table 1: Level of Anxiety

\begin{tabular}{|l|c|c|}
\hline Level of Anxiety & $\boldsymbol{f}$ & \% \\
\hline Mild $(0-21)$ & 137 & 59.67 \\
\hline Moderate (22 -35) & 57 & 24.78 \\
\hline Severe (35 up) & 36 & 15.85 \\
\hline Total & 230 & 100.00 \\
\hline
\end{tabular}

\section{Level of Fatigue}

Table 2 shows that the majority of the respondents experienced mild fatigue $(65.22 \%)$; moderate fatigue (21.74\%); severe fatigue (13.04\%).

\section{Table 2: Level of Fatigue}

\begin{tabular}{|l|c|c|}
\hline Level of Fatigue & $\boldsymbol{f}$ & $\mathbf{\%}$ \\
\hline Mild (1-3) & 150 & 65.22 \\
\hline Moderate (4-6) & 50 & 21.74 \\
\hline Severe (7-10) & 30 & 13.04 \\
\hline Total & 230 & 100.00 \\
\hline
\end{tabular}

\section{Level of Depression}

Table 3 reveals that most of the respondents experienced mild depression (49.13\%), no signs of depression or normal $(26.65 \%)$, moderate depression $(17.39 \%)$ and severe depression $(7.83 \%)$.

\section{Table 3: Depression}

\begin{tabular}{|l|c|c|}
\hline Level of Depression & $\boldsymbol{f}$ & $\mathbf{\%}$ \\
\hline Normal (0-7) & 59 & 26.65 \\
\hline Mild (8-16) & 113 & 49.13 \\
\hline Moderate (12-23) & 40 & 17.39 \\
\hline Severe (24 and above) & 18 & 7.83 \\
\hline Total & 230 & 100.00 \\
\hline
\end{tabular}

\section{DISCUSSION}

Table 1 shows the level of anxiety based on the results of Beck Anxiety Inventory Tool. Majority $(59.57 \%)$ experienced a mild level of anxiety. Mild anxiety means that the anxiety symptoms such as the feeling of coldness or thrilling sensation, feeling warm, unsteadiness of the parts of the body, uneasiness, palpitations, fear, exertion in breathing, fear of dying, stomach upsets, dizziness, flushed face and hot or cold perspirations were mildly felt by the respondents. The results of the study are congruent with the study of Mustafa et al., (2015) that majority of the students had mild to moderate levels of anxiety. Furthermore, studies have also shown that anxiety may affect the student's performance in academics which is characterized with the persistent feelings of fear, panic attacks, headaches, gastrointestinal problems, breathing difficulties, and 
weakness. Moreover, studies revealed that a number of college students have been afflicted with anxiety and that anxiety is one of the top reasons why students seek counselling (Pedrelli, 2015).

Table 2 shows the level of fatigue based on the results of the Brief Fatigue Inventory Tool. Majority (65.22\%) of the respondents experienced mild fatigue. Mild fatigue means that the respondents experienced mild level symptoms of fatigue with a rating of 0-3 in a scale of 1-10 that describes the respondents' perceived level of fatigue during the data collection, usual scale or degree of fatigue and highest scale of fatigue during the past 24 hours. Moreover, mild fatigue also means that the respondents' scale or degree of fatigue did not or just mildly restricted their activities of daily living, mood, walking stability, relationship with other people and their satisfaction with life. The same findings were reported by the studies conducted by Amaducci, Mota, \& Pimenta (2010) and Mirza \& Malik (2014) which revealed that fatigue is likely to be experienced by a person who had a prolonged mental or physical activity. Likewise, fatigue is characterized by a decreased motivation to work, reduced efficiency of accomplishment of work and is manifested by the feelings of exhaustion, drowsiness, irritability and loss of life direction.

Table 3 shows that most of the respondents (49.13\%) experienced mild depression. Mild depression means that the graduating college students experienced mild signs of depression based on the 11-item Kutcher Adolescent Depression Scale. Mild depression means that the students only seldom or even never had any of the following symptoms such as the feelings of having low mood, irritable, sleep difficulties, unworthiness, weak, unable to concentrate and, unhappiness, bothered, worried and the thoughts of taking his own life. The findings of this study support the previous studies of Naushad et al., (2014), Shamsuddin et al., 2013 and Mkize, Nonkelela, \& Mkize (1998) that revealed that majority of the students in college experienced mild to moderate level of depression and some may also experience severe depression.

\section{CONCLUSION}

Anxiety, fatigue and depression are prevalent mental health issues among college graduating students. The results of this study revealed that the majority of the respondents had mild anxiety, fatigue and depression. However, there were also some students who experienced moderate to severe anxiety, fatigue and depression. Hence, there is a need to address the issue to prevent further complications from arising. Therefore, it is recommended that health education on the prevention or management of anxiety, fatigue and depression must be given to students of colleges or universities. Likewise, students who were found to have severe levels of anxiety, fatigue and depression must be reevaluated by higher institutions to confirm the results and in order to give prompt management and treatment.

\section{Conflict of Interest}

There is no conflicting interest to be declared.

\section{ACKNOWLEDGMENT}

The authors would like to express their sincerest gratitude to the Biliran Province State University Administration as well as to the 230 research respondents for their invaluable support in the conduct of this study.

\section{REFERENCES}

Amaducci, C., Mota, D., \& Pimenta, C. (2010). Fatigue among nursing undergraduate students. Revista da Escola de Enfermagem da USP, 44(4), 1052-1058.

American Psychiatric Association (2013). Diagnostic and Statistical Manual of Mental Disorders. (5 ${ }^{\text {th }}$ edition). Arlington, Virginia: American Psychiatric Publishing.

Beck, A.T., Epstein, N., Brown, G., \& Steer, R.A. (1988). An inventory for measuring clinical anxiety: Psychometric properties. Journal of Consulting and Clinical Psychology, 56(6), 893-897.

Blanco, C., Okuda, M., Wright, C., Hasin, D.S., Grant, B.F., Liu, S.M., \& Olfson, M. (2008). Mental health of college students and their non-college-attending peers: results from the National Epidemiologic Study on Alcohol and Related Conditions. Archives of General Psychiatry, 65(12), 1429-1437.

Eisenberg, D., Hunt, J., \& Speer, N. (2013). Mental health in American colleges and universities: variation across 
student subgroups and across campuses. Journal of Nervous and Mental Disease, 201(1), 60-67.

Kessler, R.C., Amminger, G.P., Aguilar-Gaxiola, S., Alonso, J., Lee, S., \& Ustün, T.B. (2007). Age of onset of mental disorders: a review of recent literature. Current Opinion in Psychiatry, 20(4), 359-364.

LeBlanc, J., Almudevar, A., Brooks, S., \& Kutcher, S. (2002). Screening for Adolescent Depression: Comparison of the Kutcher Adolescent Depression Scale with the Beck Depression Inventory. Journal of Child and Adolescent Psychopharmacology, 12(2), 113-126.

Lee, R., Sta. Maria, M., Estanislao, S., \& Rodriguez, C. (2013). Factors Associated with Depressive Symptoms among Filipino University Students. Public Library of Science One, 8(11), e 79825.

Mirza, S., \& Malik, A. (2014). Factors associated with fatigue in clinical and non- clinical physical therapy students. Rawal Medical Journal, 39(4), 392-394.

Mkize, L., Nonkelela, N., \& Mkize, D. (1998). Prevalence of depression in a university population. Curationis, 21(3), $32-37$.

Mustafa, S., Mekonashi, E., Shkembi, F., \& Fanaj, N. (2015). Anxiety and Self-esteem among University Students: Comparison between Albania and Kosovo. Procedia-Social and Behavioral Sciences, 205, 189-194.

Naushad, S., Farooqui, W., Sharma, S., Rani, M., Singh, R., \& Verma, S. (2014). Study of proportion and determinants of depression among college students in Mangalore city. Nigerian Medical Journal: Journal of the Nigeria Medical Association, 55(2), 156-160.

Pedrelli, P., Nyer, M., Yeung, A., Zulauf, C., \& Wilens, T. (2015). College Students: Mental Health Problems and Treatment Considerations. Academic Psychiatry, 39(5), 503-511.

Shamsuddin, K., Fadzil, F., Ismail, W.S., Shah, S.A., Omar, K., Muhammad, N.A., Jaffar, A., Ismail, A., \& Mahadevan, R. (2013). Correlates of depression, anxiety, and stress among Malaysian university students. Asian Journal of Psychiatry, 6(4), 318-323.

Soyeur, F., Unalan, D., \& Elmali, F. (2010). The relationship between physical activity level and fatigue in vocational college students. International Sports Medicine Journal, 11(3), 345-355.

University of Texas MD Anderson Cancer Center (1997). Brief Fatigue Inventory (BFI). Houston, Texas: University of Texas Anderson Cancer Center. 